\title{
Defining the Optimal Management Strategy for Small Renal Masses
}

\author{
Marc Dall'Era $^{\mathrm{a}, \mathrm{b}, *}$ and Primo N. Lara, Jr. ${ }^{\mathrm{a}}$ \\ ${ }^{a}$ University of California Davis Comprehensive Cancer Center, Sacramento, CA, USA \\ ${ }^{\mathrm{b}}$ Department of Urologic Surgery, UC Davis School of Medicine, Sacramento, CA, USA
}

Received 10 May 2021

Accepted 17 May 2021

Pre-press 4 June 2021

Published 28 August 2021

Small renal masses are typically defined as solid, contrast enhancing kidney tumors under $4 \mathrm{~cm}$ in size, corresponding to American Joint Cancer Commission TNM stage T1a. They are most commonly asymptomatic and incidentally discovered, a classic by-product of increasing use of cross-sectional imaging for any variety of unrelated abdominal symptoms. The risk that a small renal mass represents a malignancy is closely tied to size with nearly $20 \%$ of tumors under $4 \mathrm{~cm}$ being benign. [1] Also, of the small renal masses that are kidney cancer, the majority have indolent histology with prolonged natural history. In this issue of the journal, Drs. Ellis and Messing provide a comprehensive review of the management of small renal masses [2]. They conclude that "short and intermediate-term data demonstrate that active surveillance with the option for delayed intervention is a safe management approach with similar survival outcomes to primary intervention (PI) at 2 and 5 years, is cost effective, and prevents overtreatment, especially in patients with significant comorbidities".

As with low-risk prostate cancer, over-detection and over-treatment of small renal masses is an important clinical problem. A 2017 study by Welch

\footnotetext{
${ }^{*}$ Correspondence to: Marc Dall'Era, MD, Professor, Department of Urologic Surgery, UC Davis School of Medicine, 4860 Y Street, Suite 3500, Sacramento, CA 95817, USA. Tel.: +1 916 734 2823; Fax: +1 916734 8094; E-mail: mdallera@ucdavis.edu.
}

et al. utilizing data from the Surveillance, Epidemiology, and End Results (SEER) program linked to Medicare claims data showed that while the incidence of kidney cancer has nearly doubled since 1975, mortality has remained relatively stagnant [3]. The authors also showed that the risk of undergoing any cross-sectional imaging was directly correlated with higher risk of undergoing a kidney procedure including nephrectomy. These data suggestnot only considerable overdiagnosis of non-lethal kidney cancers, but high risk of unnecessary treatment over time. Discovery of a small renal mass also contributes to patient fear and anxiety while incurring substantial downstream health care costs.

As with other low-grade malignancies, the first job of the treating oncologist is to help the patient overcome and manage the immediate anxiety over the word 'cancer' and realize the often-indolent nature of these tumors. As patients now have immediate access to CT scan reports, radiologists must also be aware of the impact of speculating on the histology of a small renal mass or suggesting biopsy or intervention.

Several options exist for management of a small renal mass and active surveillance should always be considered the first standard of care especially in any patient with limited life expectancy. As well described in the Ellis and Messing review [2], the outcomes of active surveillance with delayed 
intervention as needed resultin similar outcomes as primary upfront treatment. The advantage being that many patients may avoid unnecessary treatment.Percutaneous biopsy is safe and useful if the information obtained will help guide treatment decisions. Biopsy results may be particularly useful to help patients accept active surveillance even if a low-grade malignancy is found. Many patients and clinicians still select extirpative surgery for small renal masses which should be primarily partial nephrectomy to avoid risk of renal insufficiency. Most can be performed with minimally invasive laparoscopic or robotic approaches at major medical centers. Other less invasive options include percutaneous ablation using either heating or cooling technology. In experienced hands, both partial nephrectomy and percutaneous ablation can offer excellent long-term outcomes with low morbidity.

\section{ACKNOWLEDGMENTS}

The authors have no acknowledgments.

\section{FUNDING}

Supported in part by NCI Cancer Center Support Grant P30 CA093373 (PNL)

\section{AUTHOR CONTRIBUTIONS}

MD and PNL contributed to the conception of thework and interpretation of data.

\section{CONFLICT OF INTEREST}

The authors have no conflict of interest to report

\section{REFERENCES}

[1] Thompson RH, Kurta JM, Kaag M, et al. Tumor size is associated with malignant potential in renal cell carcinoma cases. J Urol. 2009; 181:2033.

[2] Ellis E, Messing E. Active Surveillance of Small Renal Masses: A Review. Kidney Cancer 2021;5(3):139-52.

[3] Welch HG, Skinner JS, Schroeck FR, et al. Regional Variation of Computed Tomographic Imaging in the United States and the Risk of Nephrectomy. JAMA Intern Med. 2018;178:221. 